\title{
STABILITY OF CONTACT METRIC MANIFOLDS AND UNIT VECTOR FIELDS OF MINIMUM ENERGY
}

\author{
D. Perrone and L. Vergori
}

\begin{abstract}
In this paper we obtain criteria of stability for $\eta$-Einstein $K$-contact manifolds, for Sasakian manifolds of constant $\varphi$-sectional curvature and for 3-dimensional Sasakian manifolds. Moreover, we show that a stable compact Einstein contact metric manifold $M$ is Sasakian if and only if the Reeb vector field $\xi$ minimises the energy functional. In particular, the Reeb vector field of a Sasakian manifold $M$ of constant $\varphi$-holomorphic sectional curvature +1 minimises the energy functional if and only if $M$ is not simply connected.
\end{abstract}

\section{INTRODUCTION}

Let $(M, g)$ be a compact Riemannian manifold. The identity map $i d_{M}:(M, g)$ $\rightarrow(M, g)$ is a harmonic map; that is, a critical point of the energy functional. Following Nagano [15], the Riemannian manifold $M$ is said to be stable if the identity map is stable, that is, the second variation of the energy functional at $i d_{M}$ is non-negative, otherwise $M$ is said to be unstable. Smith [20] proved that: if $(M, g)$ is a compact Einstein manifold of dimension $n$, then $M$ is stable if and only if $\lambda_{1} \geqslant(2 r / n)$, where $\lambda_{1}$ is the first eigenvalue of the Laplace-Beltrami operator acting on functions and $r$ is the scalar curvature. Others results on the stability of $M$ in terms of $\lambda_{1}$ can be found, for example, in [10, 21, 23, 24]. Moreover, in [20], it was proved that every holomorphic map between Kaehler manifolds is stable, in particular a compact Kaehler manifold is stable (see also [15]). Even if many concepts of Kaehlerian geometry have their correspondences in Sasakian geometry, in the theory of the harmonic maps in Sasakian geometry, and more in general in contact metric geometry, we still have few results $([4,10,14])$. From the instability theorem of Xin (see, for example, [25, p.162]) follows that the unit sphere $S^{n}, n=2 m+1 \geqslant 3$, which is a classical example of Sasakian manifold, is unstable. Concerning the study of the stability of a contact metric manifold, we know only the following result ([10, Theorem 3.1]): if $M$ is a compact Sasakian $(2 m+1)$-manifold of constant $\varphi$-sectional curvature $c \leqslant 1$ and $\lambda_{1}$ satisfies $\lambda_{1}<c(m+1)+3 m-1$, then $M$ is unstable. In [4] the authors found that:

Received 14th February, 2007

The first author was supported by funds of the MIUR (PRIN 2005)

Copyright Clearance Centre, Inc. Serial-fee code: 0004-9727/06 \$A2.00+0.00. 
a compact cosymplectic manifold (which is a particular almost contact metric manifold) is stable.

On the other hand, in the last ten years, the theory of the harmonic unit vector fields, namely the unit vector fields $U:(M, g) \rightarrow\left(T^{1} M, g_{s}\right), g_{s}$ being the Sasaki metric, which are critical points of the energy functional $E$ defined on the set $\mathfrak{X}^{1}(M)$ of all unit vector fields, has been largely developed by many authors (see, for example, $[26,29,11,16,17]$ and the references in [12]). Brito [8] proved that the Hopf vector fields on the unit sphere $S^{3}$ are the unique absolute minimisers of the energy functional $E: \mathfrak{X}^{1}\left(S^{3}\right) \rightarrow \mathbb{R}$. Wood [29] (see also [11]) proved that, for the unit sphere $S^{2 m+1}$ with $m>1$, the Hopf vector fields are unstable. We note that a Hopf vector field on $S^{2 m+1}$ is precisely the Reeb vector field $\xi$ of a natural Sasakian structure on $S^{2 m+1}$. In general, in dimension greater than three, to our knowledge, there are no examples of unit vector fields which realise an absolute minimum for the energy and neither criteria of existence of minima for the energy (see $[12,18])$.

In this paper we investigate the stability of a compact contact metric manifold $(M, g, \eta, \xi)$ in terms of the first eigenvalue $\mu_{1}$ of the Laplacian acting on 1-forms and in terms of stability of the Reeb vector field $\xi$. As a consequence of more general results, we obtain criteria of stability for $\eta$-Einstein $K$-contact manifolds (Theorem 3.1 ), for Sasakian manifolds of constant $\varphi$-sectional curvature $c$ (Theorem 3.2) and for 3dimensional Sasakian manifolds (Theorem 3.3). Finally, in Section 4 we show that a stable compact Einstein contact metric manifold $M$ is a Sasakian manifold if and only if the Reeb vector field $\xi$ minimises the energy functional $E: \mathfrak{X}^{1}(M) \rightarrow \mathbb{R}$. In particular, we get that the Reeb vector field of a compact Sasakian manifold $M$ of constant $\varphi$-holomorphic sectional curvature +1 minimises the energy functional if and only if $M$ is not simply connected (Theorem 4.2 ).

\section{THE SECOND VARIATION FORMULA}

Let $(M, g)$ and $(N, h)$ be two Riemannian manifolds with $M$ compact and ndimensional. A smooth map $f:(M, g) \rightarrow(N, h)$ is said to be harmonic if it is a critical point of the energy functional

$$
E: C^{\infty}(M, N) \rightarrow \mathbf{R}, f \mapsto E(f)=\int_{M} e(f) d v,
$$

where $e(f)=\|\mathrm{d} f\|^{2} / 2$ is the energy density and $\|\mathrm{d} f\|$ is the norm of the differential of $f$ with respect to the metrics $g$ and $h$. More precisely, $f$ is a harmonic map if $\left(\mathrm{d} E\left(f_{t}\right) / \mathrm{d}\right)_{t=0}=0$ for every smooth variation $f_{t}$ of $f$. If $f$ is a harmonic map, the Hessian form of the energy $E$ at $f$ is defined by the second variation formula [20]:

$$
(\operatorname{Hess} E)_{f}(V, V)=\left.\frac{\mathrm{d}^{2}}{\mathrm{~d} t^{2}} E\left(f_{t}\right)\right|_{t=0}=\int_{M} h\left(V, J_{f} V\right) v_{g},
$$


where $V$ is a vector field along $f$. The operator $J_{f}$, called the Jacobi operator of $f$, is a second order selfadjoint elliptic differential operator acting on the space $\Gamma\left(f^{-1} T N\right)$ of the vector fields along $f$, and is defined by

$$
J_{f} V=\bar{\Delta}_{f} V-\mathrm{Ric}_{f} V .
$$

The operator $\bar{\Delta}_{f}$, called the rough Laplacian along $f$, is defined by

$$
\bar{\Delta}_{f} V=-\sum_{i=1}^{n}\left(\bar{\nabla}_{e_{i}} \bar{\nabla}_{e_{i}} V-\bar{\nabla}_{\nabla_{e_{i} e_{i}}} V\right), \quad V \in \Gamma\left(f^{-1} T N\right),
$$

where $\bar{\nabla}$ is the connection (on the vector bundle $f^{-1} T N$ ) induced by the Levi-Civita connection of $(N, h)$, and $\left\{e_{i}\right\}_{i=1, \ldots, n}$ is a local orthonormal frame on $M$. Moreover, denoting by $R_{h}$ the curvature tensor of $(N, h)$,

$$
\operatorname{Ric}_{f} V=\sum_{i=1}^{n} R_{h}\left(f_{*} e_{i}, V\right) f_{*} e_{i} .
$$

A harmonic map $f$ is said to be stable if (Hess $E)_{f}$ is positive semi-definite, and unstable if there exists a vector field $V$ along $f$ such that (Hess $E)_{f}(V, V)<0$. The identity map $i d_{M}:(M, g) \rightarrow(M, g)$ is a trivial example of a harmonic map, but the theory of the second variation is much more complicated. From (2.1)-(2.3) we readily deduce that the second variation formula of the energy for $i d_{M}$ is given by

$$
(\operatorname{Hess} E)_{i d}(X, X)=\int_{M} g\left(J_{i d} X, X\right) v_{g}=\int_{M} g(\bar{\Delta} X-Q X, X) v_{g} \quad \forall X \in \mathfrak{X}(M),
$$

where

$$
\bar{\Delta} X=\bar{\Delta}_{i d} X=-\sum_{i=1}^{n}\left(\nabla_{e_{i}} \nabla_{e_{i}} X-\nabla_{\nabla_{e_{i}} e_{i}} X,\right.
$$

$Q$ is the Ricci operator and $J_{i d}=\bar{\Delta}-Q$ is the Jacobi operator of the identity map. The graph of the identity map is the diagonal of $M \times M$, which is a minimal submanifold. Then, Nagano [15] proved that $M$ is stable, (that is, the identity map $i d_{M}$ is stable), if and only if the diagonal in $M \times M$ is stable as minimal submanifold. In particular a compact Kaehler manifold is stable (see also [20]).

\section{STABILITY OF $\eta$-EINSTEIN CONTACT MANIFOLDS}

A $(2 m+1)$-dimensional manifold $M, m \geqslant 1$, is said to be a contact manifold if it admits a global 1-form $\eta$ such that $\eta \wedge(\mathrm{d} \eta)^{m} \neq 0$. Given $\eta$, there exists a unique vector field $\xi$, called the Reeb vector field (or the characteristic vector field) such that $\eta(\xi)=1$ and $\mathrm{d} \eta(\xi, \cdot)=0$. Furthermore, a Riemannian metric $g$ is said to be an associated metric if there exists a tensor $\varphi$ of type $(1,1)$ such that

$$
\eta=g(\xi, \cdot), \quad \mathrm{d} \eta=g(\cdot, \varphi \cdot), \quad \varphi^{2}=-\mathrm{I}+\eta \otimes \xi
$$


$(\eta, g, \xi, \varphi)$, or $(\eta, g)$, is called a contact metric structure, or contact Riemannian structure and $(M, \eta, g)$ a contact metric (or Riemannian) manifold. Let $(M, \eta, g)$ be a contact metric manifold of dimension $2 m+1, m \geqslant 1$. We denote by $\nabla$ the Levi-Civita connection, by $\rho$ the Ricci tensor, by $Q$ the corresponding Ricci operator and by $r$ the scalar curvature. The tensor $h=\mathcal{L}_{\xi} \varphi / 2$, where $\mathcal{L}$ denotes the Lie derivative, is symmetric and plays a fundamental role in contact metric geometry [2]. It satisfies the equation $([2, p .67])$

$$
\nabla \xi=-\varphi-\varphi h .
$$

The scalar torsion $\|\tau\|, \tau=\mathcal{L}_{\xi} g$, introduced in [9], and the tensor $h$ are related by $\tau=2 g(h \varphi \cdot, \cdot) . M$ is said to be $K$-contact if $\xi$ is a Killing vector field, that is, $\tau=0$, or equivalently $h=0$. Moreover $M$ is said to be a Sasakian manifold if

$$
\left(\nabla_{X} \varphi\right) Y=g(X, Y) \xi-\eta(Y) X \quad \forall X, Y \in \mathfrak{X}(M) .
$$

Any Sasakian manifold is K-contact and the converse also holds when the manifold is of dimension three. For further information about contact metric geometry we refer to [2].

From now on we denote by $\mu_{1}$ the first eigenvalue of the Laplacian $\Delta_{1}$ acting on 1-forms. We recall that $\Delta_{1}$ also acts on vector fields via duality and it is related to the rough Laplacian $\bar{\Delta}$ and the Ricci operator $Q$ by the well-known Wietzenböck's formula (see, for example, $[1$, p. 57])

$$
\Delta_{1}=\bar{\Delta}+Q
$$

The 1-form $\eta$ is an eigenform of the Laplacian $\Delta_{1}$ corresponding to the eigenvalue $4 \mathrm{~m}$. In fact, in [17] the first author proved that

$$
\bar{\Delta} \xi=4 m \xi-Q \xi
$$

hence, by (3.3),

$$
\Delta_{1} \xi=4 m \xi
$$

and so

$$
\Delta_{1} \eta=\Delta_{1} g(\xi, \cdot)=g\left(\Delta_{1} \xi, \cdot\right)=4 m g(\xi, \cdot)=4 m \eta,
$$

by which we deduce that

$$
\mu_{1} \leqslant 4 m \text {. }
$$

Now, we suppose that $M$ is a $\eta$-Einstein manifold, that is, the Ricci tensor is of the form

$$
\rho=a g+b \eta \otimes \eta \quad \text { (or equivalently } \quad Q=a \mathrm{I}+b \eta \otimes \xi)
$$

where $a, b \in C^{\infty}(M, \mathbb{R})$. It is known that for $m \geqslant 2$, on any $\eta$-Einstein K-contact manifold the functions $a$ and $b$ are constant $([2$, p. 105]). $(\kappa, \mu)$-spaces of dimension $2 m+1$, with 
$\mu=2(1-m)$, are examples of non Sasakian $\eta$-Einstein manifolds $([3$, p.206]). On a general contact metric manifold the Ricci curvature in the direction of $\xi$ is given by ([2, p. 92])

$$
\rho(\xi, \xi)=2 m-\operatorname{tr} h^{2}, \quad \operatorname{tr} h^{2}=\frac{\|\tau\|^{2}}{4} .
$$

From (3.7) we have $\rho(\xi, \xi)=a+b$ and hence

$$
a+b=2 m-\frac{\|\tau\|^{2}}{4} \text {. }
$$

Moreover, from (3.7), we have

$$
r=\operatorname{tr} \rho=(2 m+1) a+b .
$$

Consequently, we get

$$
a=\frac{r}{2 m}-1+\frac{\|\tau\|^{2}}{8 m} \text { and } \quad b=-\frac{r}{2 m}+(2 m+1)\left(1-\frac{\|\tau\|^{2}}{8 m}\right) .
$$

Assuming $M$ compact, by (2.4) and (3.3), we get

$$
\begin{aligned}
(\text { Hess } E)_{i d}(X, X)= & \int_{M} g\left(\Delta_{1} X, X\right) v_{g}-2 \int_{M} \rho(X, X) v_{g} \\
= & \int_{M} g\left(\Delta_{1} X, X\right) v_{g}-2 \int_{M}\left(\frac{r}{2 m}-1+\frac{\|\tau\|^{2}}{8 m}\right)\|X\|^{2} v_{g} \\
& \quad+2 \int_{M}\left[\frac{r}{2 m}-(2 m+1)\left(1-\frac{\|\tau\|^{2}}{8 m}\right)\right][\eta(X)]^{2} v_{g} .
\end{aligned}
$$

Now, let $\omega$ be an eigenform of $\Delta_{1}$ corresponding to the eigenvalue $\mu_{1}: \Delta_{1} \omega=\mu_{1} \omega$. Denote by $X_{0}$ the vector field dual of $\omega$. If the scalar curvature $r$ satisfies the inequality

$$
r \leqslant 2 m(2 m+1)\left(1-\frac{\|\tau\|^{2}}{8 m}\right), \text { that is } b \geqslant 0,
$$

from (3.12) we have

$$
(\operatorname{Hess} E)_{i d}\left(X_{0}, X_{0}\right) \leqslant \int_{M}\left[\mu_{1}-\left(\frac{r}{m}-2+\frac{\|\tau\|^{2}}{4 m}\right)\right]\left\|X_{0}\right\|^{2} v_{g} .
$$

Further, using (3.1), (3.7) and (3.11), we can write

$$
Q X_{0}=\left(2 m-\frac{\|\tau\|^{2}}{4}\right) X_{0}+\left[-\frac{r}{2 m}+(2 m+1)\left(1-\frac{\|\tau\|^{2}}{8 m}\right)\right] \varphi^{2} X_{0}
$$

Since

$$
g\left(\varphi^{2} X_{0}, X_{0}\right)=-g\left(\varphi^{2} X_{0}, \varphi^{2} X_{0}\right)+g\left(\varphi^{2} X_{0}, \eta\left(X_{0}\right) \xi\right)=-\left\|\varphi X_{0}\right\|^{2}
$$


if the scalar curvature satisfies the inequality

$$
r \geqslant 2 m(2 m+1)\left(1-\frac{\|\tau\|^{2}}{8 m}\right), \text { that is } \quad b \leqslant 0,
$$

by (3.14), (3.12) becomes

$$
\begin{aligned}
(\operatorname{Hess} E)_{i d}\left(X_{0}, X_{0}\right)= & \int_{M}\left[\mu_{1}-\left(4 m-\frac{\|\tau\|^{2}}{2}\right)\right]\left\|X_{0}\right\|^{2} v_{g} \\
& \quad+\int_{M}\left[-\frac{r}{m}+2(2 m+1)\left(1-\frac{\|\tau\|^{2}}{8 m}\right)\right]\left\|\varphi X_{0}\right\|^{2} v_{g} \\
\leqslant & \int_{M}\left[\mu_{1}-\left(4 m-\frac{\|\tau\|^{2}}{2}\right)\right]\left\|X_{0}\right\|^{2} v_{g}
\end{aligned}
$$

Therefore, by (3.6), (3.13) and (3.15), we obtain at once the following

Proposition 3.1. Let $(M, \eta, g)$ be a compact contact metric manifold of dimension $2 m+1$. Then, the first eigenvalue $\mu_{1}$ of the Laplacian acting on 1-forms satisfies the inequality $\mu_{1} \leqslant 4 m$. If, in addition, $M$ is $\eta$-Einstein and $\mu_{1}$ satisfies one of the following conditions:

(i) $\mu_{1}<(r / m)-2+\left(\|\tau\|^{2} / 4 m\right) \leqslant 4 m-\left(\|\tau\|^{2} / 2\right)$,

(ii) $\mu_{1}<4 m-\left(\|\tau\|^{2} / 2\right) \leqslant(r / m)-2+\left(\|\tau\|^{2} / 4 m\right)$,

then $M$ is unstable.

In [20] Smith proved that if $(M, g)$ is a compact Einstein manifold of dimension $n$, $\rho=(r / n) g$, then $M$ is stable if and only if $\lambda_{1} \geqslant(2 r / n)$, where $\lambda_{1}$ is the first eigenvalue of the Laplace-Beltrami operator $\Delta$ acting on functions. The following Proposition gives a version of Smith's result in contact metric geometry.

Proposition 3.2. Let $(M, g)$ be a compact Einstein manifold of dimension $(2 m+1)$, homothetic to a contact metric manifold $(M, \tilde{\eta}, \tilde{g})$, that is, $\widetilde{g}=\alpha g$, $\alpha=$ const $>0$. Then, the first eigenvalue $\mu_{1}$ of the Laplacian acting on 1-forms satisfies:

(i) $\mu_{1} \leqslant 4 m \alpha ;$

(ii) $\quad M$ is stable $\Leftrightarrow \mu_{1} \in\left[4 m \alpha\left(1-\left(\|\tau\|^{2} / 8 m\right)\right), 4 m \alpha\right]$, where $\|\tau\|=\left\|\mathcal{L}_{\xi} g\right\|$ is constant, $\xi=\alpha \tilde{\xi}$

(iii) if $\xi$ is Killing, $M$ is stable $\Leftrightarrow \mu_{1}=4 m \alpha$;

(iv) if $r>0, \quad \mu_{1}<4 m \alpha\left(1-\left(\|\tau\|^{2} / 8 m\right)\right) \Leftrightarrow \lambda_{1} \in[(2 m+1) \alpha(1$ $\left.\left.-\left(\|\tau\|^{2} / 8 m\right)\right), 4 m \alpha\left(1-\left(\|\tau\|^{2} / 8 m\right)\right)\right]$.

Proof: (i) We have already observed in (3.5) that the Reeb vector field $\tilde{\xi}$ is an eigenvector of the Laplacian $\widetilde{\Delta}_{1}$ corresponding to the eigenvalue $4 m: \widetilde{\Delta}_{1} \tilde{\xi}=4 m \widetilde{\xi}$. Setting $\xi=\alpha \tilde{\xi}$ we get

$$
\Delta_{1} \xi=\alpha \widetilde{\Delta}_{1} \xi=4 m \alpha^{2} \tilde{\xi}=4 m \alpha \xi
$$


and hence $\mu_{1} \leqslant 4 m \alpha$.

(ii) We first suppose that $M$ is stable. Then, by (2.4) and (3.3) we have

$$
0 \leqslant(\operatorname{Hess} E)_{i d_{M}}(X, X)=\int_{M} g\left(\Delta_{1} X-2 Q X, X\right) v_{g}
$$

for any $X \in \mathfrak{X}(M)$. Since $g$ and $\tilde{g}$ are homothetic, we have:

$$
\tilde{\rho}=\rho(X, Y)=\frac{r}{2 m+1} g=\frac{r}{(2 m+1) \alpha} \widetilde{g}
$$

that is, $(M, \widetilde{\eta}, \widetilde{g})$ is an Einstein contact metric manifold. Thus, by $(3.11),(M, \tilde{g})$ has constant scalar curvature

$$
\tilde{r}=2 m(2 m+1)\left(1-\left(\|\tau\|^{2} / 8 m\right)\right)
$$

where $r=\mathcal{L}_{\xi} g=\mathcal{L}_{\tilde{\xi}} \tilde{g}, \xi=\alpha \tilde{\xi}$. Consequently

$$
\|\tau\| \text { is constant, } \quad r=2 m(2 m+1) \alpha\left(1-\frac{\|\tau\|^{2}}{8 m}\right) \quad \text { and } \quad \rho=2 m \alpha\left(1-\frac{\|\tau\|^{2}}{8 m}\right) g .
$$

Let $X_{0}$ be a vector field eigenvector of the Laplacian $\Delta_{1}$ corresponding to the eigenvalue $\mu_{1}: \Delta_{1} X_{0}=\mu_{1} X_{0}$. From (3.16), we obtain

$$
\left[\mu_{1}-4 m \alpha\left(1-\frac{\|\tau\|^{2}}{8 m}\right)\right] \int_{M}\left\|X_{0}\right\|^{2} v_{g} \geqslant 0, \text { that is, } \mu_{1} \geqslant 4 m \alpha\left(1-\frac{\|\tau\|^{2}}{8 m}\right)
$$

and so, by (i),

$$
\mu_{1} \in\left[4 m \alpha\left(1-\frac{\|\tau\|^{2}}{8 m}\right), 4 m \alpha\right]
$$

Vice versa, we assume

$$
\mu_{1} \in\left[4 m \alpha\left(1-\frac{\|\tau\|^{2}}{8 m}\right), 4 m \alpha\right]
$$

Let $f \in C^{\infty}(M, \mathbb{R}), \mathrm{d} f \neq 0$, be an eigenfunction of the Laplace-Beltrami operator $\Delta$ corresponding to the eigenvalue $\lambda_{1}: \Delta f=\lambda_{1} f$. Then

$$
\Delta_{1}(\mathrm{~d} f)=\mathrm{d} \Delta f=\lambda_{1} \mathrm{~d} f
$$

and thus

$$
\lambda_{1} \geqslant \mu_{1} \geqslant 4 m \alpha\left(1-\frac{\|\tau\|^{2}}{8 m}\right)
$$

where $2 m \alpha\left(1-\left(\|\tau\|^{2} / 8 m\right)\right)$ is the Einstein constant of $(M, g)$ and so, by the above mentioned Smith's result, $M$ is stable.

(iii) If $\xi$ is a Killing vector field, then $\tau=0$ and so, by means of (ii), we get that $M$ is stable if and only if $\mu_{1}=4 m \alpha$. 
(iv) If $(M, g)$ has positive scalar curvature, since $(M, g)$ is Einstein with $\rho=2 m \alpha\left(1-\left(\|\tau\|^{2} / 8 m\right)\right) g$, by Lichnerowicz-Obata Theorem $\left(\left[25\right.\right.$, p. 182]) $\lambda_{1}$ satisfies the inequality

$$
\lambda_{1} \geqslant(2 m+1) \alpha\left(1-\frac{\|\tau\|^{2}}{8 m}\right) .
$$

If $\mu_{1}<4 m \alpha\left(1-\left(\|\tau\|^{2} / 8 m\right)\right)$, by the result of (ii), we get that $M$ is unstable and so (3.17) and Smith's result gives

$$
\lambda_{1} \in\left[(2 m+1) \alpha\left(1-\frac{\|\tau\|^{2}}{8 m}\right), 4 m \alpha\left(1-\frac{\|\tau\|^{2}}{8 m}\right)\right] .
$$

Of course, $\lambda_{1}<4 m \alpha\left(1-\left(\|\tau\|^{2} / 8 m\right)\right)$ implies $\mu_{1}<4 m \alpha\left(1-\left(\|\tau\|^{2} / 8 m\right)\right)$.

Next, we consider a compact $n$-dimensional Riemannian manifold $(M, g)$ and its unit tangent sphere bundle $\left(T^{\mathbf{l}} M, g_{s}\right)$ equipped with the Sasaki metric $g_{s}$. Every unit vector field $U \in \mathfrak{X}^{1}(M)$ defines a map $U:(M, g) \rightarrow\left(T^{1} M, g_{s}\right)$ and the energy of $U$ is the energy of the corresponding map $([\mathbf{2 6}, \mathbf{2 9}])$ :

$$
E(U)=\frac{1}{2} \int_{M}\|\mathrm{~d} U\|^{2} v_{g}=\frac{n}{2} \operatorname{vol}(M, g)+\frac{1}{2} \int_{M}\|\nabla U\|^{2} v_{g} .
$$

$U$ is called a harmonic vector field if it is critical for the energy functional $E: \mathfrak{X}^{1}(M) \rightarrow \mathbb{R}$. The corresponding critical point condition has been determined in $[26,29]$ and is given by:

\section{$\bar{\Delta} U$ is collinear to $U$.}

When $U$ is a harmonic vector field, the Hessian form at $U$ (of the second variation formula) takes the form $[26,11]$

$$
(\operatorname{Hess} E)_{U}(W, W)=\int_{M}\left(\|\nabla W\|^{2}-\|W\|^{2}\|\nabla U\|^{2}\right) v_{g}
$$

where $W \in U^{\perp}$. $U$ is called stable if (Hess $\left.E\right)_{U}(W, W) \geqslant 0$ for any $W \in U^{\perp}$, otherwise it is said to be unstable.

If $(M, \eta, g)$ is a contact metric manifold, in [17] was proved that $\xi$ is a harmonic vector field if and only if it is an eigenvector of the Ricci operator. In such case $M$ is called an $H$-contact manifold. We now suppose that $(M, \eta, g)$ is a compact $\eta$-Einstein contact manifold. From (3.7) it follows that $M$ is $H$-contact. If $\xi$ is unstable, then there exists a vector field $W \in$ Ker $=\xi^{\perp}$ such that

$$
(\operatorname{Hess} E)_{\xi}(W, W)=\int_{M}\left(\|\nabla W\|^{2}-\|W\|^{2}\|\nabla \xi\|^{2}\right) v_{g}<0
$$

Since $\|\xi\|=1$, we obtain $g(\bar{\Delta} \xi, \xi)=\|\nabla \xi\|^{2}$. Therefore, by (3.4) and (3.8), we have

$$
\|\nabla \xi\|^{2}=4 m-\rho(\xi, \xi)=2 m+\frac{\|\tau\|^{2}}{4}
$$


and (3.19) gives

$$
\int_{M}\left[\|\nabla W\|^{2}-\left(2 m+\frac{\|\tau\|^{2}}{4}\right)\|W\|^{2}\right] v_{g}<0 .
$$

Then, by (2.4) and (3.20), we obtain

$$
\begin{aligned}
(\operatorname{Hess} E)_{i d}(W, W) & =\int_{M}\left[\|\nabla W\|^{2}-\rho(W, W)\right] v_{g} \\
& <\int_{M}\left[\left(2 m+\frac{\|\tau\|^{2}}{4}\right)\|W\|^{2}-\rho(W, W)\right] v_{g} .
\end{aligned}
$$

Using (3.7), since $W \in \operatorname{Ker} \eta$, we have $\rho(W, W)=a\|W\|^{2}$. Consequently, (3.21) becomes

$$
(\operatorname{Hess} E)_{i \mathrm{~d}}(W, W)<\int_{M}\left[2 m+1-\frac{r}{2 m}+(2 m-1) \frac{\|\tau\|^{2}}{8 m}\right]\|W\|^{2} v_{g} .
$$

From (3.22), we get the following

PROPOSITION 3.3. Let $M$ be a compact $\eta$-Einstein contact manifold of dimension $(2 m+1)$, with scalar curvature

$$
r \geqslant 2 m(2 m+1)+(2 m-1) \frac{\|\tau\|^{2}}{4} .
$$

If $\xi$ is unstable, then $M$ is unstable.

Boyer, Galicki and Matzeu [6] study $\eta$-Einstein geometry as a class of distinguished Riemannian metrics on contact metric manifolds. In particular they show that there are many compact $\eta$-Einstein Sasakian manifolds. We recall that a compact $\eta$-Einstein K-contact manifold with scalar curvature $r>-2 m$ is Sasakian (see Boyer and Galicki [5], or [2, p. 106]). Moreover, on any $\eta$-Einstein K-contact manifold the functions $a$ and $b$ and the scalar curvature $r$ are constant. Then, Propositions 3.1, 3.2 and 3.3 imply the following

THEOREM 3.1. Let $(M, \eta, g)$ be a compact $\eta$-Einstein $K$-contact manifold of dimension $2 m+1, m>1$.

(1) If $\mu_{1}<\min \{(r / m)-2,4 m\}$, then $M$ is an unstable $\eta$-Einstein Sasakian manifold.

(2) If $r \geqslant 2 m(2 m+1)$ and $\xi$ is unstable, then $M$ is an unstable $\eta$-Einstein Sasakian manifold.

(3) If $r=2 m(2 m+1)$, then $M$ is Sasaki-Einstein and

(i) $\mu_{1}=4 m \Leftrightarrow M$ is stable,

(ii) $\mu_{1}<4 m \Leftrightarrow(2 m+1) \leqslant \lambda_{1}<4 m$. 
The unit sphere $S^{2 m+1}$ is an example of Sasakian space of constant sectional curvature +1 . We denote by $\left(\eta_{0}, g_{0}\right)$ the standard Sasakian structure on $S^{2 m+1}$. Let $(M, g)$ be an odd dimensional compact Riemannian manifold of constant sectional curvature +1 . Then, $(M, g)$ is a spherical space form $\left(S^{2 m+1} / \Gamma, g\right)$, where $\Gamma$ is a finite subgroup of $O(2 m+2)$ in which only the identity element has +1 as an eigenvalue, and $g$ is the Riemannian metric on the quotient space $S^{2 m+1} / \Gamma$ induced by $g_{0}$. The unitary group $U(m+1)$ can be considered as the subgroup of all elements of $O(2 m+2)$ which preserve $\eta_{0}$, and $\Gamma$ is conjugate in $O(2 m+2)$ to a subgroup of $U(m+1)$, then $M$ inherits a contact 1-form $\eta$ from $\eta_{0}$ (Wolf [28]). Since the metric $g$ is induced from $g_{0}$, then $(\eta, g)$ is a Sasakian structure on the spherical space form $M$ (Tanno [22, p. 505]). So, we have that: every odd-dimensional compact Riemannian manifold of constant sectional curvature +1 is a Sasakian manifold. This fact corrects a remark of Gherghe, Ianus and Pastore ([10, p. 51]). Now, we consider a Sasakian space form $M^{2 m+1}(c)$, that is, a $(2 m+1)$-dimensional Sasakian manifold of constant $\varphi$-sectional curvature $c$. Such spaces have constant scalar curvature $r=(m / 2)[(2 m+1)(c+3)+c-1]$, and are $\eta$-Einstein ([2, p. 113]) with

$$
a=\frac{(m+1) c+3 m-1}{2} \text { and } b=\frac{(m+1)(1-c)}{2} .
$$

Moreover, $c=+1$ if and only if $M^{2 m+1}$ has constant sectional curvature +1 . On the other hand, Urakawa ([24, p. 572]) proved that the first eigenvalue $\lambda_{1}$ of the Laplace-Beltrami operator on $\left(S^{2 m+1} / \Gamma, g\right), \Gamma \neq\left\{i d_{M}\right\}$, is bigger than or equal to $4 m$, that is, $\left(S^{2 m+1} / \Gamma, g\right)$, $\Gamma \neq\left\{i d_{M}\right\}$, is stable. Therefore, these results and Theorem 3.1 yield

TheOREM 3.2. Let $(M, \eta, g)$ be a compact Sasakian space form of constant $\varphi$-sectional curvature $c, \operatorname{dim} M=2 m+1 \geqslant 3$.

(i) If $\mu_{1}<\min \{(m+1) c+3 m-1,4 m\}$, then $M$ is unstable.

(ii) If $c \geqslant 1$ and $\xi$ is unstable, then $M$ is unstable.

(iii) If $c=1$, then: $\mu_{1}=4 m \Leftrightarrow M$ is stable $\Leftrightarrow$ the fundamental group $\pi_{1}(M) \neq\{0\}$.

In Theorem 3.2 we can also consider the case $m=1$ because in such case $M$ is $\eta$-Einstein and the functions $a=(c+1)$ and $b=(1-c)$ are constant.

REMARK 3.1 .

(a) In [10, Theorem 3.1], the authors considered a Sasakian space form $M^{2 m+1}(c)$ with $c \leqslant 1$ and proved that $\lambda_{1}<(m+1) c+3 m-1$ implies that $M$ is unstable. Of course, our Theorem 3.2(i) extends and improves such result.

(b) We recall that $\mu_{1}\left(S^{2 m+1}\right)=2 m+1$ and $\mu_{2}\left(S^{2 m+1}\right)=4 m$. Then, if $M$ is a compact Sasakian manifold of constant curvature +1 , with $\pi_{1}(M) \neq\{0\}$, from Theorem 3.2 we deduce that $\mu_{1}(M)=\mu_{2}\left(S^{2 m+1}\right)$. 
(c) By (3.21) we easily deduce that, if $(M, \eta, g)$ is a compact K-contact $(2 m$ $+1)$-manifold with Ricci tensor $\rho \geqslant 2 m g$, then the instability of the Reeb vector field implies the instability of $M$.

The results given in Theorems 3.1 and 3.2 can be sharpened in dimension three. In fact, any $K$-contact three-manifold is Sasakian and $\eta$-Einstein. More precisely the Ricci tensor is given by

$$
\rho=\left(\frac{\tau}{2}-1\right) g+\left(-\frac{r}{2}+3\right) \eta \otimes \eta
$$

Then, by the same arguments used in the proofs of Theorems 3.1 and 3.2, we deduce the following

THEOREM 3.3. Let $(M, \eta, g)$ be a compact Sasakian manifold of dimension three. If holds one of the following conditions

(i) $\mu_{1}<r-2 \leqslant 4$,

(ii) $\mu_{1}<4 \leqslant r-2$,

(iii) $r \geqslant 6$ and $\xi$ is unstable,

then $M$ is a unstable. Moreover, if $r=6$, then: $\mu_{1}=4 \Leftrightarrow M$ is stable $\Leftrightarrow \pi_{1}(M) \neq\{0\}$.

\section{EXISTENCE OF MINIMA FOR THE ENERGY $E_{\mid x^{1}(M)}$}

Brito [8] proved that the Hopf vector fields on the unit sphere $S^{3}$, that is, the unit vector fields tangent to the fiber of any Hopf fibration, are the unique minimisers of the energy functional $E: \mathfrak{X}^{1}\left(S^{3}\right) \rightarrow \mathbb{R}$. In particular they are stable. On the other hand, $S^{3}$ is unstable, so the converse of Theorem 3.3 (iii) is not true. However, the Hopf vector fields on the unit sphere $S^{2 m+1}, m>1$, are unstable $([29,11])$. Besides, to our knowledge, in dimension $>3$, there are no examples of unit vector fields which realise an absolute minimum for the energy and neither criteria of existence of minima for the energy (see $[12,18])$. Theorems of Section 3 give results for which the instability of the Reeb vector field implies the instability of the contact metric manifold, or, equivalently, the stability of the contact metric manifold gives the stability of the Reeb vector field. So, it is natural to study the existence of minima for the energy $E_{\mid \mathfrak{X}^{1}(M)}$ when $M$ is a stable compact Riemannian manifold. Urakawa [23] classified all the compact simply connected irreducible Riemannian symmetric spaces which are stable (such spaces are in particular Einstein).

In this section we discuss the existence of minima for the energy $E: \mathfrak{X}^{1}(M) \rightarrow \mathbb{R}$, where $(M, g)$ is supposed to be a stable compact Einstein manifold of dimension $n, \rho=\kappa g$. Since $M$ is stable, by (2.4), we have

$$
\begin{aligned}
(\operatorname{Hess} E)_{i d}(X, X) & =\int_{M} g(\bar{\Delta} X-Q X, X) v_{g} \\
& =\int_{M}\left(\|\nabla X\|^{2}-\rho(X, X)\right) v_{g} \geqslant 0 \quad \forall X \in \mathfrak{X}(M) .
\end{aligned}
$$


Then the energy $E: \mathfrak{X}^{1}(M) \rightarrow \mathbb{R}$, given by (3.18), satisfies

$$
E(U)=\frac{1}{2} \int_{M}\|\nabla U\|^{2} v_{g}+\frac{n}{2} \operatorname{vol}(M, g) \geqslant \frac{\kappa+n}{2} \operatorname{vol}(M, g) \quad \forall U \in \mathfrak{X}^{1}(M) .
$$

Now, we recall that a vector field $X$ is called to be a Jacobi vector field if it is a solution of the equation $J_{i d} X=0$. Jacobi vector fields are studied in [30] and there labelled "geodesic vector fields". Then, if $U_{0}$ is a unit Jacobi vector field, we have

$$
\int_{M}\left\|\nabla U_{0}\right\|^{2} v_{g}=\int_{M} g\left(\bar{\Delta} U_{0}, U_{0}\right) v_{g}=\int_{M} \rho\left(U_{0}, U_{0}\right) v_{g}=\kappa \operatorname{vol}(M, g) .
$$

Hence,

$$
E\left(U_{0}\right)=\frac{\kappa+n}{2} \operatorname{vol}(M, g) \leqslant E(U) \quad \forall U \in \mathfrak{X}^{1}(M),
$$

that is, $U_{0}$ minimises the energy. Vice versa, let $U$ be a unit vector field such that

$$
E(U)=\frac{\kappa+n}{2} \operatorname{vol}(M, g) \text {. }
$$

Then, by (4.2), $\int_{M}\|\nabla U\|^{2} v_{g}=\kappa \operatorname{vol}(M, g)$, and thus, by (4.1), we get

$$
(\operatorname{Hess} E)_{i d}(U, U)=0 .
$$

Since $M$ is stable, we can expand $U$ into the infinite sum:

$$
U=\sum_{i=1}^{\infty} E_{i}, \quad \text { with } \quad J_{i d} E_{i}=a_{i} E_{i} \quad \text { and } \int_{M} g\left(E_{i}, E_{j}\right) v_{g}=0 \quad \forall i \neq j \text {, where } a_{i} \geqslant 0 .
$$

Then, we have

$$
J_{i d} U=\sum_{i=p+1}^{\infty} a_{i} E_{i}, \quad \text { where } p=\operatorname{dim} \operatorname{Ker} J_{i d} \quad \text { and } a_{i}>0 \quad \forall i \geqslant p+1,
$$

and thus

$$
0=(\operatorname{Hess} E)_{i d}(U, U)=\int_{M} g\left(J_{i d} U, U\right) v_{g}=\sum_{i=p+1}^{\infty} a_{i} \int_{M} g\left(E_{i}, E_{i}\right) v_{g}
$$

implies that $E_{i}=0$ for any $i \geqslant p+1$. So, $J_{i d} U=0$, that is, $U$ is a Jacobi vector field. If $U \in \mathfrak{X}^{1}(M)$ is a unit vector field whose flow is volume-preserving, that is, $\operatorname{div} U=0$, then it is easy to get that $U$ is a Killing vector field if and only if $U$ is a Jacobi vector field (see, for example, $[19$, p. 171]). Hence we have

PROPOSITION 4.1. Let $(M, g)$ be a stable compact Einstein manifold. Then, the unit Jacobi vector fields are the unique unit vector fields which minimise the energy. In particular, if $U \in \mathfrak{X}^{1}(M)$ is volume-preserving, then $U$ minimises the energy if and only if $U$ is a Killing vector field. 
Moreover, we obtain

THEOREM 4.1. Let $(M, \eta, g)$ be a stable compact Einstein contact metric manifold. Then, the Reeb vector field $\xi$ minimises the energy if and only if $M$ is a Sasakian manifold.

Proof: First, we note that the flow of the Reeb vector field $\xi$ of a contact metric manifold is volume-preserving. In fact, by (3.2), choosing a (local) orthonormal basis $E_{i}$ of eigenvectors of the tensor $h$, we have

$$
\operatorname{div} \xi=\sum_{i=1}^{2 m} g\left(\nabla_{E_{i}} \xi, E_{i}\right)=\sum_{i=1}^{2 m} g\left(-\varphi E_{i}-\varphi h E_{i}, E_{i}\right)=0 .
$$

Besides, by a result of Boyer-Galicki [5], a compact Einstein $K$-contact manifold is Sasaki-Einstein. Then, the result follows from Proposition 4.1.

Finally, we obtain the following result.

THEOREM 4.2. Let $(M, \eta, g)$ be a compact Sasakian manifold of constant sectional curvature $+1, \operatorname{dim} M=2 m+1, m>1$. Then, the Reeb vector field $\xi$ minimises the energy if and only if $M$ is not simply connected.

Proof: We first show that if $\xi$ minimises the energy, then necessarily $M$ is not simply connected. In fact, if $M$ were simply connected, then $M$ would be the unit sphere $S^{2 m+1}$. On the other hand, on the sphere $S^{2 m+1}$ the Hopf vector fields are exactly the unit Killing vector fields [27]. So, $\xi$ would be a Hopf vector field and we know that Hopf vector fields on $S^{2 m+1}, m>1$, are unstable. This would imply that $\xi$ does not minimise the energy, that is, a contradiction. The converse follows from Theorems 3.2 and 4.1.

REMARK 4.1. (On the volume functional) Let $(M, g)$ be a compact Riemannian manifold and $U \in \mathfrak{X}^{1}(M)$. Then, $U$ determines a map between $(M, g)$ and $\left(T^{1} M, g_{s}\right)$ and $\operatorname{vol}(U)$ is the volume of the corresponding submanifold $U(M)$ of $\left(T^{1} M, g_{s}\right) . U$ is said to be minimal if it is a critical point of the functional vol: $\mathfrak{X}^{\mathbf{1}}(M) \rightarrow \mathbf{R}$. The study of the volume of unit vector fields begins with the pioneering work of Gluck-Ziller [13], where the motivation was to find the optimal unit vector fields on the unit sphere $S^{3}$. They proved that unit vector fields of minimum volume on $S^{3}$ are precisely the Hopf vector fields, equivalently the unit Killing vector fields, and no others. For more details and the state of the art for the minimality of unit vector fields, we can refer to the survey [12]. The Reeb vector field $\xi$ of a compact Sasakian manifold is minimal, concerning its stability Borrelli [7] proved that if $\xi$ is E-stable, that is, stable with respect to the energy, then there exists a number $\left.\left.k_{s} \in\right] 0, \infty\right]$, called stability number, such that the vector field $\tilde{\xi}=\sqrt{k} \xi \in \mathfrak{X}^{1}(M, \tilde{g}=(1 / k) g)$ is stable with respect to the volume if and only if $0<k \leqslant k_{s}$. However, his paper does not contain examples of Sasakian manifolds with $\xi$ E-stable. Applying Theorem 4.2 and the result of Borrelli, we obtain that: if $(M, \eta, g)$ is a compact Sasakian manifold of constant sectional curvature +1 , 
$\operatorname{dim} M=2 m+1, m>1$ and $\pi_{1}(M) \neq\{0\}$, then there exists $\left.\left.k_{s} \in\right] 0, \infty\right]$, such that the vector field $\tilde{\xi}=\sqrt{k} \xi \in \mathfrak{X}^{1}(M, \tilde{g}=(1 / k) g)$ is stable with respect to the volume if and only if $0<k \leqslant k_{\mathrm{a}}$.

\section{REFERENCES}

[1] A. Besse, Einstein manifolds (Springer-Verlag, Berlin, 1987).

[2] D.E. Blair, Riemannian geometry of contact and symplectic manifold, Progress in Math. 203 (Birkhäuser, Boston, Basel, Berlin, 2002).

[3] D.E. Blair, T. Koufogiorgos and B.J. Papantoniou, 'Contact metric manifolds satisfying a nullity condition', Israel J. Math. 91 (1995), 189-214.

[4] E. Boeckx and C. Gherge, 'Harmonic maps and cosymplectic manifolds', J. Austral. Math. Soc. 78 (2004), 75-92.

[5] C.P. Boyer and K. Galicki, 'Einstein manifolds and contact geometry', Proc. Amer. Math. Soc. 129 (2001), 2419-2430.

[6] C.P. Boyer, K. Galicki and P. Matzeu, 'On eta-Einstein Sasakian geometry', Comm. Math. Phys. 262 (2006), 177-208.

[7] V. Borrelli, 'Stability of the Reeb vector field of a Sasakian manifold', Soochow J. Math. 30 (2004), 283-292.

[8] F.B. Brito, 'Total bending of flows with mean curvature correction', Differential Geom. Appl. 12 (2000), 157-163.

[9] S.S. Chern and R.S. Hamilton, 'On Riemannian metrics adapted to three-dimensional contact manifolds', in Lect. Notes in Math. 1111 (Springer-Verlag, Berlin, Heidelberg, New York, 1985), pp. 279-305.

[10] C. Gherghe, S. Ianus and A.M. Pastore, 'CR-manifolds, harmonic maps and stability', J. Geom. 71 (2001), 42-53.

[11] O. Gil-Medrano and E. Llinares-Fuster, 'Second variation of volume and energy of vector fields. Stability of Hopf vector fields', Math. Ann. 320 (2001), 531-545.

[12] O. Gil-Medrano, 'Unit vector fields that are critical points of the volume and of the energy: characterization and examples', in Complex, Contact and Symmetric Manifolds, (O. Kowalski, E. Musso, D. Perrone, Editors), Progress in Math. 234 (Birkhäuser, Boston, Basel, Berlin, 2005), pp. 165-186.

[13] H. Gluck and W. Ziller, 'On the volume of a unit vector field on the three sphere', Comment. Math. Helv. 61 (1986), 177-192.

[14] S. Ianus and A.M. Pastore, 'Harmonic maps on contact metric manifolds', Ann. Math. Blaise Pascal 2 (1995), 43-53.

[15] T. Nagano, 'Stability of harmonic maps between symmetric spaces', in Harmonic Maps, Lecture Notes in Math. 948 (Springer-Verlag, Berlin and New York, 1982).

[16] D. Perrone, 'Harmonic characteristic vector fields on contact metric three-manifolds', Bull. Austral. Math. Soc. 67 (2003), 305-315.

[17] D. Perrone, 'Contact metric manifolds whose characteristic vector field is a harmonic vector field', Differential Geom. Appl. 20 (2004), 367-378.

[18] D. Perrone, 'Stability of the Reeb vector field of a $H$-contact manifold', (preprint 2006).

[19] W.A. Poor, Differential geometric structures (McGraw Hill, New York, 1981). 
[20] R.T. Smith, 'The second variation formula for harmonic mappings', Proc. Amer. Math. Soc. 47 (1975), 229-236.

[21] J. Takahashi, 'On the gap between the first eigenvalue of the Laplacian on functions and 1-forms', J. Math. Soc. Japan 53 (2001), 307-319.

[22] S. Tanno, 'Sasakian manifolds with constant $\phi$-holomorphic sectional curvature', Tohoku Math. J. 16 (1993), 171-180.

[23] H. Urakawa, "The first eigenvalue of the Laplacian for a positively curved homogeneous Riemannian maniolds', Compositio Math. 59 (1986), 57-71.

[24] H. Urakawa, 'Stability of harmonic maps and eigenvalues of the Laplacian', Trans. Amer. Math. Soc. 301 (1987), 557-589.

[25] H. Urakawa, Calculus of variations and harmonic maps, Transl. Math. Monogr. 132 (Amer. Math. Soc., Providence, R.I., 1993).

[26] G. Wiegmink, 'Total bending of vector fields on Riemannian manifolds', Math. Ann. 303 (1995), 325-344.

[27] G. Wiegmink, 'Total bending of vector fields on the sphere $S^{3}$, Differential Geom. Appl. 6 (1996), 219-236.

[28] J.A. Wolf, 'A contact structure for odd dimensional spherical spaces forms', Proc. Amer. Math. Soc. 19 (1968), 196.

[29] C.M. Wood, 'On the energy of a unit vector field', Geom. Dedicata 64 (1997), 319-330.

[30] K. Yano and T. Nagano, 'On geodesic vector fields in a compact orientable Riemannian space', Comm. Math. Helv. 35 (1961), 55-64.

Dipartimento di Matematica "Ennio De Giorgi"

Università di Lecce, 73100

Lecce

Italy

e-mail: domenico.perrone@unile.it

luigi.vergori@unile.it 